\title{
Coupling of Photonic and Plasmonic Modes in Oxide and Supported Metal Nanoparticles: Finite Element Simulation and EELS Study
}

\author{
Yifan Wang $^{1}$ and Peter Crozier ${ }^{2}$
}

${ }^{1}$ School for the Engineering of Matter, Transport and Energy, Arizona State University, United States, ${ }^{2}$ Arizona State University, Tempe, Arizona, United States

Solar-driven photocatalytic water splitting offers an attractive pathway for the generation of non-carbonaceous renewable fuels (e.g., $\mathrm{H}_{2}$ ). Novel photocatalytic materials are needed to efficiently harvest the energy present in the visible region of the incident solar irradiation. Photocatalysts based on ceria $\left(\mathrm{CeO}_{2}\right)$ nanocubes, which have a bandgap of $3.2 \mathrm{eV}$, can absorb and trap sub-bandgap photons through cavity mode resonances, offering a possible solution to achieve enhanced visible light absorption [1]. Many semiconductor photocatalysts are loaded with metal co-catalyst nanoparticles to enhance the transfer of photo-generated charge carriers and to improve the catalytic reaction kinetics. Interestingly, excited cavity modes may couple with certain modes in the supported metal (e.g., surface plasmon in Ag), which has the potential for catalyzing photoreactions and realizing the conversion of solar into chemical energy. A fundamental understanding of the coupling processes is necessary to fully utilize it for photocatalytic chemistry. Monochromated electron energy-loss spectroscopy (EELS) in an aberration-corrected scanning transmission electron microscope (AC STEM) is a powerful tool to investigate the coupling between photonic and plasmonic modes on oxide-supported metal nanoparticles [2]. In addition to providing high spatial and energy resolution, the electron beam can also simultaneously excite and detect the optical and plasmonic modes as well as any potential coupling between them [3].

We conducted aloof-beam EELS simulations prior to performing any EELS experiments in order to investigate the feasibility of experimentally detecting mode coupling on a variety of $\mathrm{CeO}_{2}$-supported metal nanoparticles. Simulations were performed in COMSOL Multiphysics ${ }^{\circledR}$, which provides the numerical solution from Maxwell equations through the finite element analysis. Figure 1 shows a schematic view of a typical simulation setting. The electron beam is set $10 \mathrm{~nm}$ away from the cube to avoid the bulk signal. A metal (e.g., $\mathrm{Ag}, \mathrm{Ni}$, etc.) hemisphere is attached to the opposite face center of a ceria cube. The dielectric data of ceria is obtained from Järrendahl et al. [4]. Simulations on the cube itself with length of $250 \mathrm{~nm}$ have shown a good agreement with published experimental data [2].

Figure 2 displays a series of aloof-beam spectra where silver has been selected as the supported metal. We obtained the dielectric data of Ag from Yang et al. [5]. Figure 2(a) shows the spectra from an isolated ceria cube, a ceria cube with an $\mathrm{Ag}$ hemisphere $75 \mathrm{~nm}$ in radius, and an isolated $\mathrm{Ag}$ hemisphere $75 \mathrm{~nm}$ in radius. A blueshift and intensity increase are seen to occur at multiple peaks as indicated by the black arrows. The peak shift and intensity changes are not the results of the simple superposition of the ceria and Ag spectrum, which indicates a possible coupling effect. In Figure 2(b), we focused on the peaks between $3-3.5 \mathrm{eV}$ and investigated the effect of increasing the Ag hemisphere radius from 60 to $90 \mathrm{~nm}$. With the size of the Ag hemisphere increasing, the energy of the major peak shows a blueshift trend, while there is also an increase in intensity. Also, the minor peak at higher energy shows a trend of broadening. The evidence of possible coupling between the photonic modes of the ceria and the plasmons of the silver will be investigated experimentally with a Cs-corrected STEM. Further simulations and experiments on other metals are also expected. 

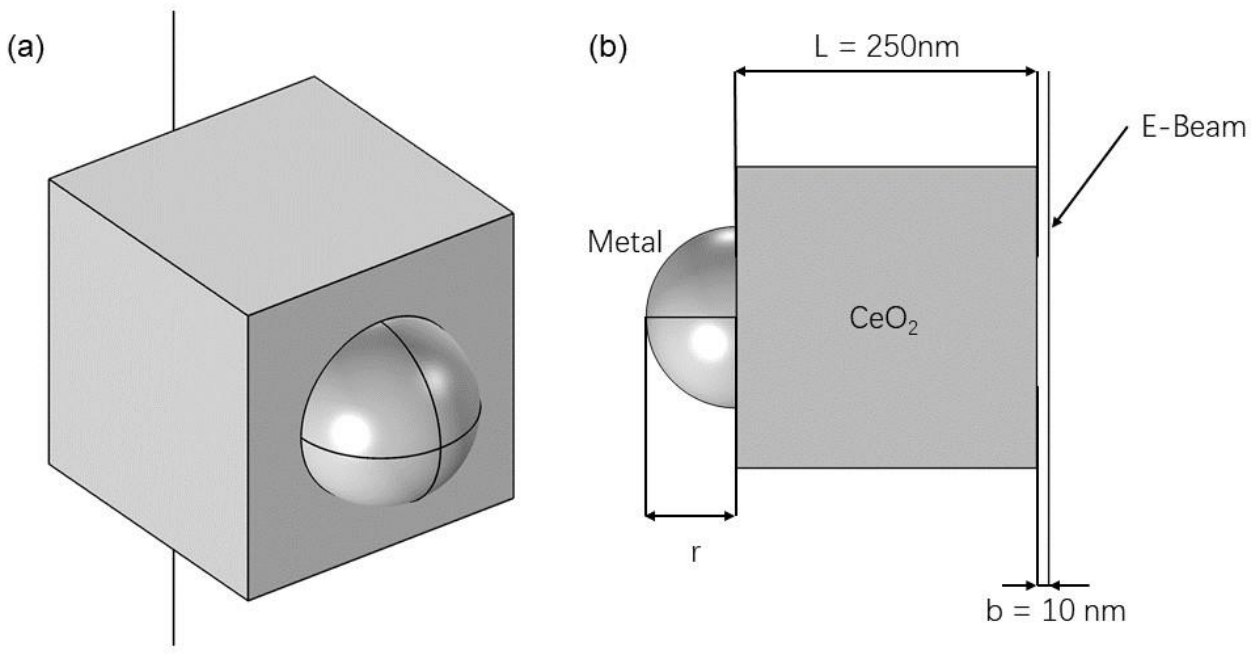

Figure 1. Figure 1. Schematic view of metal-loaded $\mathrm{CeO} 2$ nanocube aloof-beam EELS simulations. (a) The $3 \mathrm{D}$ view of the simulation geometry, with the electron beam depicted as a solid line. (b) Side view of the scheme with size and parameters. The side length, $\mathrm{L}$, of the ceria cube is $250 \mathrm{~nm}$ and the impact parameter of the electron beam, b, is $10 \mathrm{~nm}$. The radius, $\mathrm{r}$, of the metal hemisphere ranges from $25 \mathrm{~nm}$ to $100 \mathrm{~nm}$.

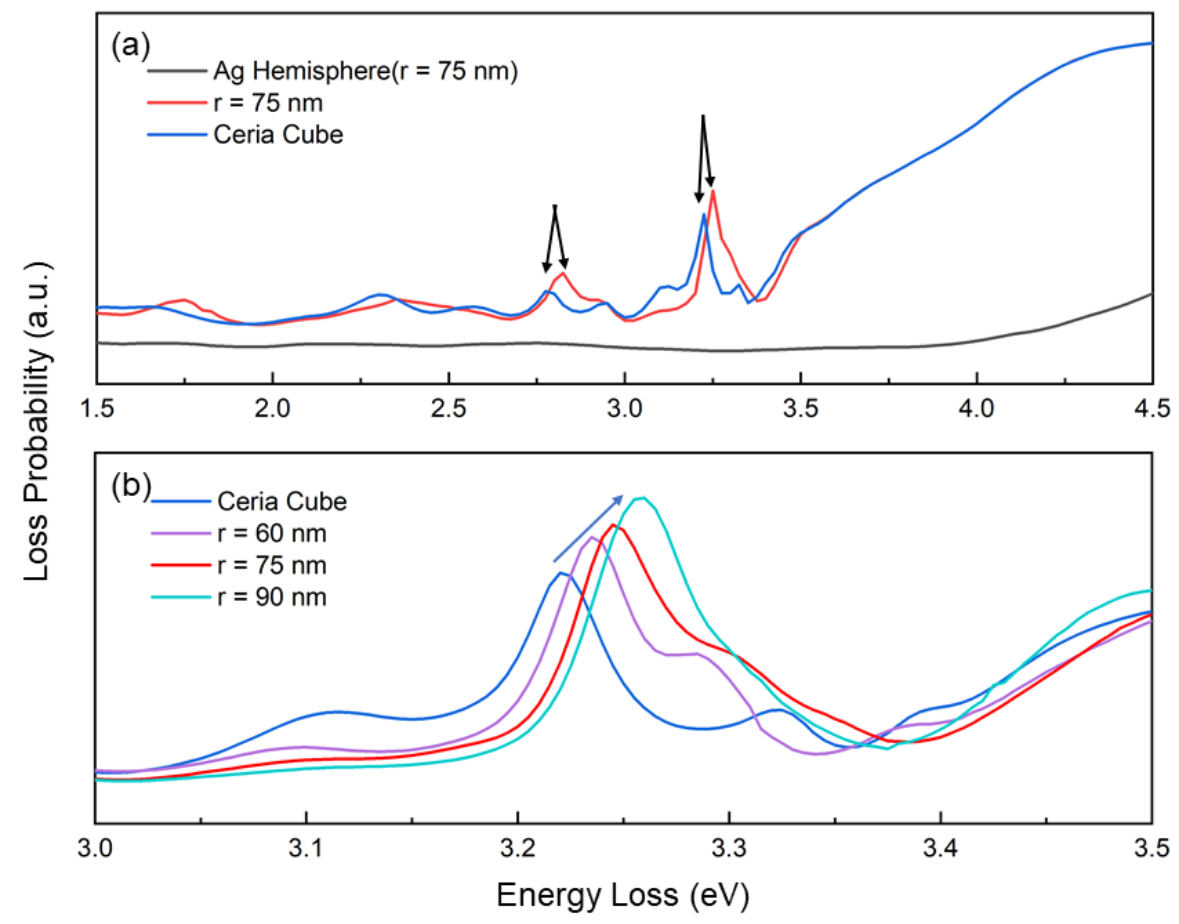

Figure 2. Figure 2. Simulated spectra of ceria cube and silver hemisphere. (a) The spectra from an energy-loss ranging from $1.5 \mathrm{eV}-4.5 \mathrm{eV}$. Peak blueshift and intensity increase from pure ceria (blue curve) to ceria cube 
attached with Ag hemisphere (red curve) can be observed at the two peaks indicated by the black arrows. The energy loss from the pure Ag hemisphere is also shown (black curve). (b) The detailed spectra in energy range $3.0-3.5 \mathrm{eV}$. As the radius of hemisphere increases, a peak shift from $3.22 \mathrm{eV}$ (blue) to $3.26 \mathrm{eV}$ (cyan) is observed

\section{References}

[1] G. S. Blaustein, M. I. Gozman, O. Samoylova, I. Y. Polishchuk, and A. L. Burin, "Guiding optical modes in chains of dielectric particles," Opt. Express, vol. 15, no. 25, p. 17380, 2007, doi: 10.1364/OE.15.017380.

[2] Q. Liu, S. C. Quillin, D. J. Masiello, and P. A. Crozier, "Nanoscale probing of resonant photonic modes in dielectric nanoparticles with focused electron beams," Phys. Rev. B, vol. 99, no. 16, p. 165102, Apr. 2019, doi: 10.1103/PhysRevB.99.165102.[3] F. J. García De Abajo, "Optical excitations in electron microscopy," Rev. Mod. Phys., vol. 82, no. 1, pp. 209-275, 2010, doi: 10.1103/RevModPhys.82.209.

[4] K. Järrendahl and H. Arwin, "Multiple sample analysis of spectroscopic ellipsometry data of semitransparent films," Thin Solid Films, vol. 313-314, pp. 114-118, 1998, doi: 10.1016/S0040-6090(97)007815.

[5] H. U. Yang, J. D'Archangel, M. L. Sundheimer, E. Tucker, G. D. Boreman, and M. B. Raschke, "Optical dielectric function of silver," Phys. Rev. B - Condens. Matter Mater. Phys., vol. 91, no. 23, pp. 1-11, 2015, doi: 10.1103/PhysRevB.91.235137.

[6] The support from U.S. DOE (BES DE-SC0004954) is gratefully acknowledged. 